\title{
The Preoperative Controlling Nutritional Status Score Predicts Survival After Curative Surgery in Patients with Pathological Stage I Non-small Cell Lung Cancer
}

\author{
FUMIHIRO SHOJI, NAOKI HARATAKE, TAKAKI AKAMINE, SHINKICHI TAKAMORI, MASAKAZU KATSURA, \\ KAZUKI TAKADA, GOUJI TOYOKAWA, TATSURO OKAMOTO and YOSHIHIKO MAEHARA \\ Department of Surgery and Science, Graduate School of Medical Sciences, Kyushu University, Fukuoka, Japan
}

\begin{abstract}
Background: The prognostic Controlling Nutritional Status (CONUT) score is used to evaluate immuno-nutritional conditions and is a predictive factor of postoperative survival in patients with digestive tract cancer. We retrospectively analyzed clinicopathological features of patients with pathological stage I non-small cell lung cancer (NSCLC) to identify predictors or prognostic factors of postoperative survival and to investigate the role of preoperative CONUT score in predicting survival. Patients and Methods: We selected 138 consecutive patients with pathological stage I NSCLC treated from August 2005 to August 2010. We measured their preoperative CONUT score in uni- and multivariate Cox regression analyses of postoperative survival. Results: A high CONUT score was positively associated with preoperative serum carcinoembryonic antigen level $(p=0.0100)$ and postoperative recurrence $(p=0.0767)$. In multivariate analysis, the preoperative CONUT score [relative risk $(R R)=6.058 ; 95 \%$ confidence interval $(C I)=1.068-113.941 ; \quad p=0.0407)$, increasing age $(R R=7.858 ; 95 \% \quad C I=2.034-36.185$; $p=0.0029)$, and pleural invasion $(R R=36.615 ; 95 \%$ $C I=5.900-362.620 ; p<0.0001)$ were independent prognostic factors. In Kaplan-Meier analysis of recurrence-free survival (RFS), cancer-specific survival (CS), and overall survival $(O S)$, the group with high CONUT score had a significantly shorter RFS, CS, and OS than did the low-CONUT score group by log-rank test ( $p=0.0458, p=0.0104$ and $p=0.0096$,
\end{abstract}

Correspondence to: Fumihiro Shoji, MD, Ph.D., Department of Surgery and Science, Graduate School of Medical Sciences, Kyushu University, 3-1-1 Maidashi, Higashi-ku, Fukuoka 812-8582, Japan. Tel: +81 926425466, Fax: +81 926425482, e-mail: fshoji@surg2.med.kyushuu.ac.jp

Key Words: Pathological stage I non-small cell lung cancer (NSCLC), preoperative Controlling Nutritional Status (CONUT) score, predictive factor, survival. respectively). Conclusion: The preoperative CONUT score is both a predictive and prognostic factor in patients with pathological stage I NSCLC. This immuno-nutritional score can indicate patients at high risk of postoperative recurrence and death.

Lung cancer is the leading cause of death from cancer worldwide. One of the reasons for this is that the outcomes of surgical resection of early-stage lung cancer remain worse than those of other malignancies. A total of $10-20 \%$ of patients with pathological stage I non-small cell lung cancer (NSCLC) experience relapse and die even after undergoing curative surgery (1).

In pathological stage IA NSCLC, several pathological factors, such as vessel invasion, including intratumoral blood vessel (BVI) and lymphatic vessel (LVI) invasion $(2,3)$, and greater tumor size $(4,5)$, have been reported as prognostic factors. Moreover, 5-year survival of patients with primary tumors larger than $2.0 \mathrm{~cm}$ is lower (5). In Japanese patients, especially those with pathological stage IA NSCLC primary tumors of 2 to $3 \mathrm{~cm}$, uracil-tegafur (UFT) treatment tends to improve survival (6). However, adjuvant chemotherapy has recently been shown not to significantly lengthen survival of patients with pathological stage IA NSCLC $(6,7)$. Additionally, the prognosis of patients with pathological stage IB NSCLC is not satisfactory, although postoperative adjuvant chemotherapy using UFT has been established in Japan. Therefore, identifying new predictors of prognosis in pathological stage I NSCLC are required and could lead to improved prognosis.

Several studies have reported that preoperative nutritional and immunological status are associated not only with postoperative complications, but also with long-term outcomes of patients with malignant tumors (8-10). A study recently reported that systemic inflammation, nutritional status, and the immune microenvironment of the tumor in lung cancer appeared to be related to prognosis of surgically resected lung cancer (11). The Controlling Nutritional Status (CONUT) 
Table I. Clinical profiles of patients.

\begin{tabular}{lc}
\hline & $\mathrm{N}(\%)$ or median (range) \\
\hline Total assessable patients & $138(100)$ \\
Follow-up, months & $58(0-94)$ \\
Age, years & $68(37-86)$ \\
Gender & $59(42.8)$ \\
Female & $79(57.2)$ \\
$\quad$ Male & \\
Smoking status & $50(36.2)$ \\
$\quad$ Never & $88(63.8)$ \\
Former & \\
Preoperative CEA level (mg/ml) & $81(58.7)$ \\
$\quad$ Normal & $57(41.3)$ \\
Abnormal & $128(92.8)$ \\
Preoperative CYFRA level (mg/ml) & $10(7.2)$ \\
$\quad$ Normal & \\
Abnormal & $97(70.3)$ \\
Surgical procedure & $41(29.7)$ \\
$\quad$ Lobectomy & \\
Limited resection & $15(10.9)$ \\
Adjuvant chemotherapy & $123(89.1)$ \\
$\quad$ Yes & No
\end{tabular}

CEA, Carcinoembryonic antigen; CYFRA, cytokeratin 19 fragment.

score is calculated based on three parameters, including serum albumin concentration, total lymphocyte count and total cholesterol concentration in peripheral blood (12). The serum albumin concentration is used as an indicator of protein reserves. The total lymphocyte count is used as an indicator of impaired immune defense. The serum total cholesterol concentration is used as a parameter of caloric depletion. Therefore, this score represents three important immunonutritional indices. The CONUT score is an efficient tool for early detection and continuous control of undernutrition in the hospital, and allows assessment of nutritional status in all inpatients (13). The CONUT score is widely used as an objective tool for nutritional assessment in inflammatory disease, chronic heart failure, and chronic liver disease (1418). Recently, the CONUT score was shown to be a predictive or prognostic marker for malignancies, such as colorectal and esophageal cancer $(19,20)$. However, the usefulness of the CONUT score for lung cancer is still unknown.

We hypothesized that the CONUT score plays a role as a prognostic factor in lung cancer. Thus, this study aimed to investigate the role of the preoperative CONUT score in survival of patients with pathological stage I NSCLC.

\section{Patients and Methods}

Patients. This study was approved by the Ethics Committee of the Kyushu University Hospital (no. 27-261). Written informed consent for the comprehensive use of clinicopathological materials was also
Table II. Assessment of the patients' nutritional status using the preoperative score.

\begin{tabular}{lcccc}
\hline Variable & \multicolumn{4}{c}{ Degree of undernutrition } \\
\cline { 2 - 5 } & Normal & Light & Moderate & Severe \\
\hline Serum albumin & & & & \\
$\quad$ Concentration $(\mathrm{g} / \mathrm{dl})$ & $\geq 3.5$ & $3.0-3.49$ & $2.50-2.99$ & $<2.50$ \\
Score & 0 & 2 & 4 & 6 \\
Total lymphocyte & & & & \\
Count $\left(/ \mathrm{mm}^{3}\right)$ & $\geq 1600$ & $1200-1599$ & $800-1199$ & $<800$ \\
Score & 0 & 1 & 2 & 3 \\
Total cholesterol & & & & \\
Concentration $(\mathrm{mg} / \mathrm{dl})$ & $\geq 180$ & $140-179$ & $100-139$ & $<100$ \\
Score & 0 & 1 & 2 & 3 \\
\hline
\end{tabular}

obtained from all patients. From August 2005 to August 2010, 391 consecutive patients with primary lung cancer underwent complete surgical resection at the Department of Surgery and Science, Graduate School of Medical Sciences, Kyushu University. One hundred and thirty-eight patients had pathological stage I NSCLC and remaining 253 patients had pathological stage II-III NSCLC. For this study, we selected 138 of those patients with pathological stage I NSCLC who had preoperative measurement of the three immuno-nutritional parameters of serum lymphocyte count, serum albumin concentration, and serum total cholesterol concentration. Their clinical profiles are shown in Table I. These parameters were determined in follow-up examinations occurring over a median period of 58 months (range=0-94 months) after surgical resection. Postoperative follow-up consisted of chest computed tomography (CT), abdominal CT, bone scintigraphy, and brain magnetic resonance imaging (MRI) at 6-month intervals during the first year, and yearly thereafter. Chest roentgenography and blood tests that included tumor markers were checked at 3- or 4-month intervals during the first year and at 6-month intervals thereafter. The study group included 59 women and 79 men, with a mean age at surgery of 68 years (range $=37-86$ years). Fifty $(36.2 \%)$ patients had never smoked and the remaining 88 patients were former or current smokers. Fifty-seven $(41.3 \%)$ patients had abnormal preoperative carcinoembryonic antigen (CEA) level (normal value: $<3.4 \mathrm{ng} / \mathrm{ml}$ ) and $10(7.2 \%)$ patients had abnormal preoperative cytokeratin 19 fragment level (normal value: $<3.5 \mathrm{ng} / \mathrm{ml}$ ). Ninety-seven $(70.3 \%$ ) patients underwent lobectomies with systemic lymphadenectomy and 41 patients underwent limited resection, including segmentectomies or wedge resections in those with peripheral lesions or poor pulmonary function. Fifteen (10.9\%) patients received adjuvant chemotherapy. Postoperative recurrence occurred in $23(16.7 \%)$ patients. Postoperative recurrence, such as local and distant recurrence, was defined as in a previous report (21): the first appearance of any new lesion suspected to be recurrence of the original lung cancer was defined as postoperative recurrence. Postoperative recurrence was clinically diagnosed by combination of CT, MRI, a bone scintigram, and fluorodeoxyglucose positronemission tomography (FDG-PET), or was pathologically diagnosed if necessary. Local recurrence was defined as that occurring in the hilar or mediastinal lymph nodes, pleural cavity, bronchial stump, or staple line. All other sites, including the brain, lung, adrenal 
glands, bone, and other locations, were considered as distant recurrences. Clinically equivocal cases were excluded in this study as second primary cancer.

Preoperative calculation of the CONUT score and determination of the cut-off value. Preoperative blood samples were obtained within 2 weeks before surgery. The preoperative CONUT score was calculated using the serum albumin concentration, peripheral lymphocyte count, and total cholesterol concentration (Table II) (12). The sum of the individual scores was defined as the CONUT score. The receiver operating characteristics (ROC) curve for the preoperative CONUT score was analyzed, and cancer-specific survival (CS) was predicted by comparing the area under the ROC curve (AUC). We decided that the best cut-off value for the preoperative CONUT score was 1 (sensitivity $=91.67 \%$; specificity= 46.07\%; AUC of the ROC curve=0.703). Seventy-nine (57.2\%) patients had a preoperative CONUT score of 1 or more (high CONUT score) and the remaining $59(42.8 \%)$ patients had a preoperative CONUT score of 0 (low CONUT score).

Histopathological evaluation. We retrospectively collected formalinfixed and paraffin-embedded NSCLC surgical specimens and reviewed them as hematoxylin-eosin-stained sections. Elastic and connective tissues were stained to determine pleural invasion, and intratumoral BVI and LVI. BVI and LVI were distinguished by Elastica van Giessen staining. A specimen was considered positive for intratumoral vessel invasion when cancer cells were observed in the intratumoral vessel lumen. Patients' pathological stages were based on the TNM classification of the International Union Against Cancer (22). For TNM staging, all of the patients underwent CT scans of the thorax and the upper abdomen, bone scintigrams, and brain CT, MRI, or FDG-PET. The histological type was adenocarcinoma in $105(76.1 \%)$ patients and non-adenocarcinoma in $33(23.9 \%)$ patients. Further details are given in Table III).

Statistical analysis. Categorical variables were analyzed using Fisher's exact test. Continuous variables were analyzed using two-sided tests. Recurrence-free survival (RFS) was defined as the interval between resection and the first recurrence event. CS was calculated from the time of resection to the date of death from lung cancer. Overall survival (OS) was calculated from the time of resection to the date of death from any cause. We analyzed patient survival using the KaplanMeier method and compared groups using the log-rank test. Uni- and multivariate analyses were performed using a logistic regression model. We used the Cox proportional hazards model to identify independent predictive and prognostic factors. A value of $p<0.05$ was considered significant. All statistical analyses were performed using JMP software, version 10.0 (SAS Institute Inc., NC, USA).

\section{Results}

Correlation between patient characteristics and preoperative CONUT score. A high CONUT score was significantly associated with abnormal preoperative serum CEA level $(p=0.0100)$ and postoperative recurrence $(p=0.00767)$. A high CONUT score was not associated with other factors, including age, sex, smoking status, histology, preoperative serum CYFRA level, pathological T factor, pleural invasion, BVI, nor LVI (Table IV).
Table III. Pathological profiles of patients.

\begin{tabular}{lc}
\hline & $\mathrm{N}(\%)$ \\
\hline Histological type & \\
Adenocarcinoma & $105(76.1)$ \\
Non-adenocarcinoma & $33(23.9)$ \\
Tumor size & \\
T1a $(\leq 2 \mathrm{~cm})$ & $59(42.8)$ \\
T1b $(>2 \mathrm{~cm})$ & $33(23.9)$ \\
T2a & $46(33.3)$ \\
Pleural invasion (pl) & \\
p10 & $118(85.5)$ \\
p11-2 & $20(14.5)$ \\
Intratumoral blood vessel invasion & \\
Without & $104(75.4)$ \\
With & $34(24.6)$ \\
Intratumoral lymphatic vessel invasion & \\
Without & $131(94.9)$ \\
With & $7(5.1)$ \\
\hline
\end{tabular}

Prognostic factors in patients with pathological stage I NSCLC. We compared CS for the following variables: older than 75 years versus younger, male versus female, past or current smokers versus never-smokers, abnormal versus normal preoperative serum CEA level, abnormal versus normal preoperative serum CYFRA levels, high versus low preoperative CONUT score, T2a pathological $\mathrm{T}$ factor versus $\mathrm{T} 1 \mathrm{ab}$, non-adenocarcinoma versus adenocarcinoma, limited resection versus lobectomy, BVI versus no BVI, LVI versus no LVI, and adjuvant chemotherapy versus no adjuvant chemotherapy (Table V). Univariate analysis showed that older age $(p=0.0002)$, history of smoking $(p=0.03771)$, abnormal preoperative serum CEA level $(p=0.0014)$, abnormal preoperative CONUT score $(p=0.0049)$, T2a pathological $\mathrm{T}$ factor $(p=0.0088)$, and pleural invasion $(p<0.0001)$ significantly affected CS. In multivariate analysis, preoperative CONUT score of 1 or more [relative risk $(\mathrm{RR})=6.058 ; 95 \%$ confidence interval $(\mathrm{CI})=1.068-113.941 ; \quad p=0.0407)$, age over 75 years ( $\mathrm{RR}=7.858 ; 95 \% \mathrm{CI}=2.034-36.185 ; p=0.0029)$, and pleural invasion $(\mathrm{RR}=36.615 ; 95 \% \mathrm{CI}=5.900-362.620 ; p<0.0001)$ were independent prognostic factors of poorer survival (Table V).

Preoperative CONUT score and RFS, CS, and OS in patients with pathological stage I NSCLC. In Kaplan-Meier analysis of RFS, CS, and OS by the preoperative CONUT score, the groups with a high CONUT score had a significantly shorter RFS, CS and OS than did the group with a low CONUT score 5-year RFS $=78.94 \%$ vs. 90.71\%, $p=0.0458$, Figure 1A; 5-year CS=88.64\% vs. 98.28\%, $p=0.0104$, Figure 1B; 5-year $\mathrm{OS}=77.49 \%$ vs. $91.10 \%, p=0.0096$, Figure 1C). 
Table IV. Patient characteristics based on the preoperative Controlling Nutritional Status (CONUT) score.

\begin{tabular}{|c|c|c|c|}
\hline Variable & $\begin{array}{c}\text { Low CONUT } \\
\text { score }(\mathrm{n}=59)\end{array}$ & $\begin{array}{c}\text { High CONUT } \\
\text { score }(\mathrm{n}=79)\end{array}$ & $p$-Value \\
\hline Age & & & 0.1798 \\
\hline$>75$ Years & 10 & 21 & \\
\hline$\leq 75$ Years & 49 & 58 & \\
\hline Gender & & & 0.1891 \\
\hline Male & 30 & 49 & \\
\hline Female & 29 & 30 & \\
\hline Smoking history & & & 0.5612 \\
\hline Current/former & 36 & 52 & \\
\hline Never & 23 & 27 & \\
\hline Histology & & & 0.6547 \\
\hline Non-adenocarcinoma & 13 & 20 & \\
\hline Adenocarcinoma & 46 & 59 & \\
\hline Preop CEA level & & & 0.0100 \\
\hline Abnormal & 17 & 40 & \\
\hline Normal & 42 & 39 & \\
\hline Preop CYFRA level & & & 0.3750 \\
\hline Abnormal & 3 & 7 & \\
\hline Normal & 56 & 72 & \\
\hline Pathological T factor & & & 0.3383 \\
\hline $\mathrm{T} 2 \mathrm{a}$ & 17 & 29 & \\
\hline T1ab & 42 & 50 & \\
\hline Pleural invasion & & & 0.4469 \\
\hline $\mathrm{pl1-2}$ & 8 & 12 & \\
\hline pl0 & 51 & 67 & \\
\hline $\begin{array}{l}\text { Intratumoral blood } \\
\text { vessel invasion }\end{array}$ & & & 0.3168 \\
\hline Yes & 12 & 22 & \\
\hline No & 47 & 57 & \\
\hline $\begin{array}{l}\text { Intratumoral lymphatic } \\
\text { vessel invasion }\end{array}$ & & & 0.4259 \\
\hline Yes & 4 & 3 & \\
\hline No & 55 & 76 & \\
\hline Postop recurrence & & & 0.0767 \\
\hline Yes & 6 & 17 & \\
\hline No & 53 & 62 & \\
\hline
\end{tabular}

Preop, Preoperative; CEA, carcinoembryonic antigen; CYFRA, cytokeratin 19 fragment; Postop: postoperative.

\section{Discussion}

In the present study, we attempted to identify new predictor or prognostic factors from a different perspective, the immuno-nutritional aspect, in addition to the pathological approach (2-5). Our study showed several novel findings. Firstly, approximately $57 \%$ of patients had a high preoperative CONUT score, which indicated malnutrition. However, all patients appeared to be sufficiently healthy to undergo surgical resection based on their appearance or results of preoperative functional tests. This finding indicates that the preoperative CONUT score can be used to select patients with undernutrition among those having adequate organ function to undergo surgery.
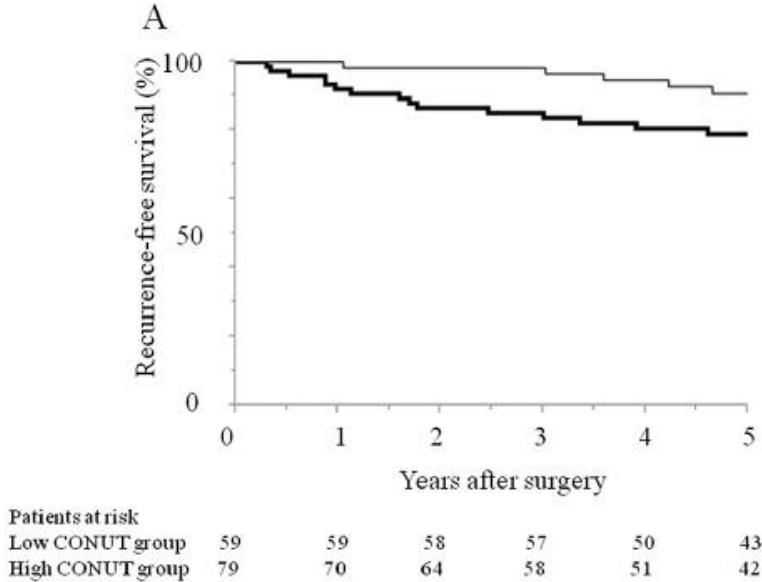

$\begin{array}{lllllll}\text { High CONUT group } & 79 & 70 & 64 & 58 & 51 & 42\end{array}$
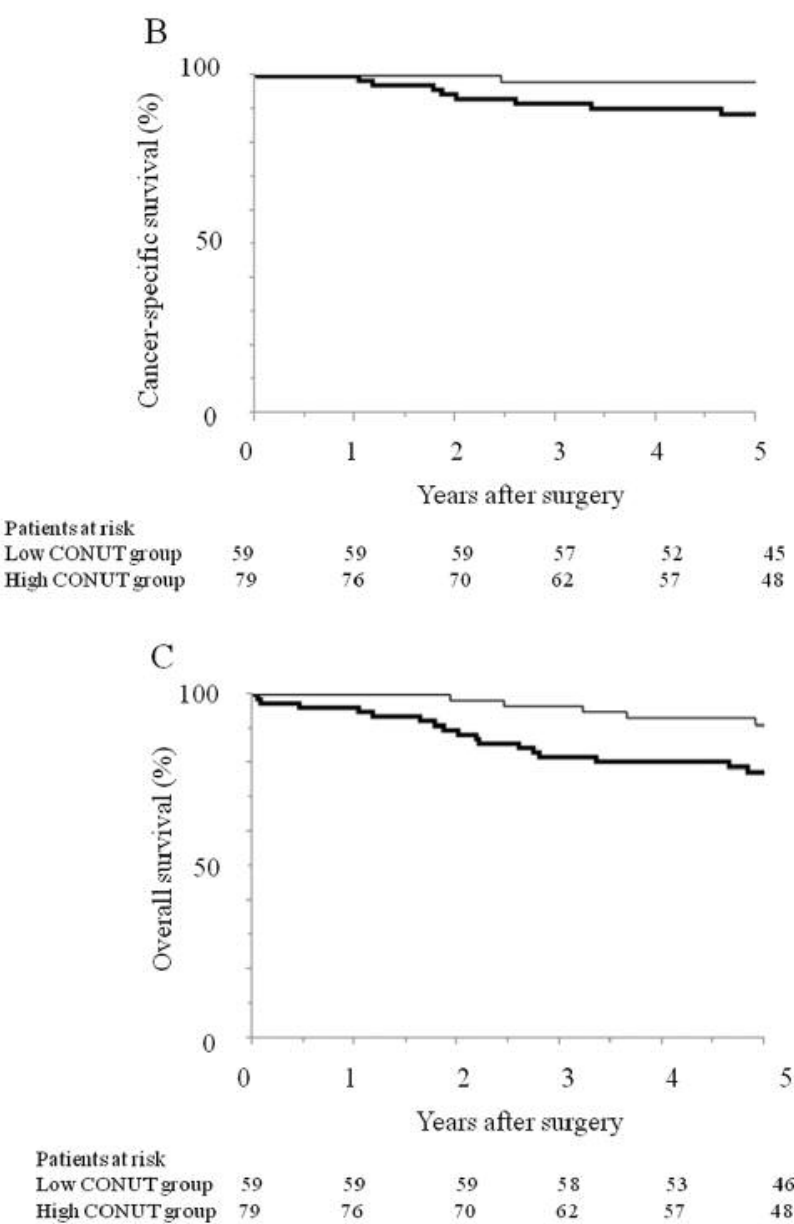

Figure 1. Kaplan-Meier curve analysis of recurrence-free (RFS) (A), cancer-specific $(C S)(B)$ and overall survival $(O S)(C)$ for 138 patients with stage I non-small cell lung cancer by preoperative Controlling Nutritional Status (CONUT) score. Thick line: High CONUT score group; thin line: low CONUT score group. The group with high CONUT score had a significantly shorter RFS, CS, and OS than did the group with a low CONUT score (5-year RFS $=78.94 \%$ vs. 90.71\%, $p=0.0458$; 5 -year CS $=88.64 \%$ vs. $98.28 \%, p=0.0104 ; 5$-year $O S=77.49 \%$ vs. $91.10 \%, p=0.0096$, log-rank test). 
Table V. Univariate and multivariate analyses for cancer-free survival in patients with stage I non-small cell lung cancer.

\begin{tabular}{|c|c|c|c|c|}
\hline \multirow[b]{2}{*}{ Variable } & \multicolumn{2}{|c|}{ Univariate analysis } & \multicolumn{2}{|c|}{ Multivariate analysis } \\
\hline & $\mathrm{RR}(95 \% \mathrm{CI})$ & $p$-Value & $\mathrm{RR}(95 \% \mathrm{CI})$ & $p$-Value \\
\hline Age: $>75 v s . \leq 75$ years & $9.828(3.051-37.275)$ & 0.0002 & $7.858(2.034-36.185)$ & 0.0029 \\
\hline Sex: Male $v s$. female & $1.697(0.534-6.362)$ & 0.3771 & - & \\
\hline Smoking status: Curr/former vs. never & $1.449(0.453-5.468)$ & 0.0377 & $2.646(0.537-12.892)$ & 0.2228 \\
\hline Preop CEA: Abnormal vs. normal & $7.973(2.098-51.912)$ & 0.0014 & $2.519(0.588-17.487)$ & 0.2275 \\
\hline Preop CYFRA: Abnormal vs. normal & $1.310(0.071-7.005)$ & 0.8049 & - & \\
\hline Preop CONUT score: High $v s$. low & $9.066(1.762-165.680)$ & 0.0049 & $6.058(1.068-113.941)$ & 0.0407 \\
\hline Tumor size: T2a $v s . \mathrm{T} 1 \mathrm{ab}$ & $4.699(1.475-17.649)$ & 0.0088 & $1.371(0.180-8.589)$ & 0.7430 \\
\hline Histology: Non-Ad vs. Ad & $1.802(0.480-5.736)$ & 0.3552 & - & \\
\hline Procedure: Limited $v s$. lobectomy & $1.930(0.569-6.082)$ & 0.2759 & - & \\
\hline Pleural invasion: Yes $v s$. no & $21.376(5.679-103.372)$ & $<0.0001$ & $36.615(5.900-362.620)$ & $<0.0001$ \\
\hline BVI: Yes $v s$. no & $2.765(0.795-9.199)$ & 0.1054 & - & \\
\hline LVI: Yes vs. no & $2.032(0.111-10.638)$ & 0.539 & - & \\
\hline Adjuvant chemotherapy: No vs. yes & $1.269(0.224-23.769)$ & 0.8191 & - & \\
\hline
\end{tabular}

95\% CI: 95\% Confidence interval; curr/former: current/former smoker; preop: preoperative; CEA, carcinoembryonic antigen; CYFRA, cytokeratin 19 fragment; CONUT: controlling nutritional status; limited: limited resection; Ad: adenocarcinoma; BVI: intratumoral blood vessel invasion; LVI: lymphatic vessel invasion; RR: relative risk.

Secondly, a high preoperative CONUT score, advanced age, and the pleural invasion were independent prognostic factors in patients with pathological stage I NSCLC by multivariate analysis. Iseki et al. evaluated the prognostic significance of the preoperative CONUT score in patients with colorectal cancer (19). The 5-year CS rate was significantly higher in the low CONUT group (score $\leq 2$ ) compared with the high CONUT group (score $\geq 3$ ). Multivariate analysis showed that the CONUT score was an independent risk factor for CS in patients who underwent curative surgery for colorectal cancer. Hirahara et al. also suggested that the CONUT score was a significant predictor of CS in patients with esophageal cancer (20). In multivariate analysis, the CONUT score was independently associated with a worse prognosis in patients undergoing curative resection for esophageal cancer. Moreover, they showed that the CONUT score was a significant predictor of $\mathrm{CS}$ in patients with surgically resected esophageal cancer. Our results strongly support these data. In addition, as far as we are aware of, the present study is the first to report that a high preoperative CONUT score was a poor prognostic factor in stage I NSCLC. Thirdly, RFS, CS, and OS in patients with stage I NSCLC with a high CONUT score were significantly shorter than those in patients with a low CONUT score. Therefore, the high-CONUT score group is a high-risk group, even after curative surgery for pathological stage I NSCLC. These results suggest that the CONUT score is a predictive and prognostic factor of postoperative recurrence and survival, and could be used to indicate high-risk patients with pathological stage I NSCLC. Patients in the high-CONUT score group may need further therapy, such as induction therapy or adjuvant therapy, and require more careful follow-up after surgery.

Fourthly, our study also shows that an elevated preoperative CEA level and postoperative recurrence were associated with a high CONUT score. Therefore, a high CONUT score might indicate progression of the tumor and suggests a high grade of tumor malignancy.

We previously reported that the preoperative prognostic nutritional index (PNI) is a simple and novel predictor of postoperative recurrence in pathological stage I NSCLC (23). PNI was calculated by two parameters: total lymphocyte count in peripheral blood and serum albumin concentration (24). Thus, PNI appears to reflect both the immune response to cancer cells and nutritional status (23). On the other hand, the CONUT score was calculated using three parameters: total lymphocyte count in peripheral blood, serum albumin concentration and serum total cholesterol concentration. A low serum cholesterol level is related to a poor prognosis in patients with several types of malignancies, such as colorectal cancer, renal cell cancer, and pancreatic cancer (25-27). Moreover, hypocholesterolemia is likely to be a secondary event after systemic inflammation (20). Cengiz et al. suggested that hypocholesterolemia is induced by cancer (25). The CONUT score enables evaluation of the protein reserve, calorie depletion, and immune defense. Consequently, the CONUT score can reflect immunological and nutritional status, and may be a good immuno-nutritional marker. In addition, the CONUT score is a simple assessment for nutritional status because it is calculated only from laboratory data. However, there are few data available regarding the utility of the clinical application of the CONUT score in organ 
malignancies, apart from colorectal or esophageal cancer (19, 20). Thus, we investigated the effects of the preoperative CONUT score on survival of patients with pathological stage I NSCLC. As a result, we found that the CONUT score was a predictive and prognostic factor of postoperative recurrence and survival in patients with pathological stage I NSCLC. However, this study has some limitations. It was a retrospective study from a single institution. A multicenter prospective study is required to evaluate the criteria of the CONUT score used in this study and the survival benefit of multimodality therapies against pathological stage I NSCLC in patients with a high preoperative CONUT score.

In conclusion, the preoperative CONUT score is a both predictive and prognostic factor in patients with pathological stage I NSCLC. This immuno-nutritional score can highlight patients with pathological stage I NSCLC who will need careful follow-up. In addition, multimodality therapies, such as induction, adjuvant chemotherapy or pre/post surgery nutritional support, might be beneficial for patients with pathological stage I NSCLC with a high preoperative CONUT score.

\section{References}

1 Sawabata N, Miyaoka E, Asamura H, Nakanishi Y, Eguchi K, Mori M, Nomori H, Fujii Y, Okumura M and Yokoi K; Japanese Joint Committee for Lung Cancer Registration: Japanese Lung Cancer Registry study of 11,663 surgical cases in 2004: demographic and prognosis changes over decade. J Thorac Oncol 6: 1229-1235, 2011.

2 Goldstein NS, Mani A, Chmielewski G, Welsh R and Pursel S: Prognostic factors in T1N0M0 adenocarcinomas and bronchioloalveolar carcinomas of the lung. Am J Clin Pathol 112: 391-402, 1999.

3 Shoji F, Haro A, Yoshida T, Ito K, Morodomi Y, Yano T and Maehara Y: Prognostic significance of intratumoral blood vessel invasion in pathologic stage IA non-small cell lung cancer. Ann Thorac Surg 89: 864-869, 2010.

4 Christian C, Erica S and Uliano M: The prognostic impact of tumor size in resected stage I non-small cell lung cancer: evidence for a two thresholds tumor diameters classification. Lung Cancer 54: 185-191, 2006.

5 Chang MY, Mentzer SJ, Colson YL, Linden PA, Jaklitsch MT, Lipsitz SR and Sugarbaker DJ: Factors predicting poor survival after resection of stage IA non-small cell lung cancer. J Thorac Cardiovasc Surg 134: 850-856, 2007.

6 Kato $\mathrm{H}$, Ichinose $\mathrm{Y}$, Ohta $\mathrm{M}$, Hata E, Tsubota N, Tada H, Watanabe Y, Wada H, Tsuboi M, Hamajima N and Ohta M; Japan Lung Cancer Research Group on Postsurgical Adjuvant Chemotherapy: A randomized trial of adjuvant chemotherapy with uracil-tegafur for adenocarcinoma of the lung. N Engl $\mathbf{J}$ Med 350: 1713-1721, 2004.

7 Pignon JP, Tribodet H, Scagliotti GV, Douillard JY, Shepherd FA, Stephens RJ, Dunant A, Torri V, Rosell R, Seymour L, Spiro SG, Rolland E, Fossati R, Aubert D, Ding K, Waller D and Le Chevalier T; LACE Collaborative Group: Lung adjuvant cisplatin evaluation: a pooled analysis by the LACE collaborative group. J Clin Oncol 26: 3552-3559, 2008.
8 Lien YC, Hsieh CC, Wu YC, Hsu HS, Hsu WH, Wang LS, Huang $\mathrm{MH}$ and Huang BS: Preoperative serum albumin level is a prognostic indicator for adenocaarcinoma of the gastric cardia. J Gastointest Surg 8: 1041-1048, 2004.

9 Ray-Coquard I, Cropet C, Van Glabbeke M, Sebban C, Le Cesne A, Judson I, Tredan O, Verweij J, Biron P, Labidi I, Guastalla JP, Bachelot T, Perol D, Chabaud S, Hogendoorn PC, Cassier P, Dufresne A and Blay JY; European Organization for Research and Treatment of Cancer Soft Tissue and Bone Sarcoma Group: Lymphopenia as a prognostic factor for overall in advanced carcinomas, sarcomas, and lymphomas. Cancer Res 69: 53835391, 2009.

10 Schwegler I, von Holzen A, Gutzwiller JP, Schlumpf R, Muhlebach S and Stanga Z: Nutritional risk is a clinical predictor of postoperative mortality and morbidity in surgery for colorectal cancer. Br J Surg 97: 92-97, 2010.

11 Alifano M , Mansuet-Lupo A, Lococo F, Roche N, Bobbio A, Canny E, Schussler O, Dermine H, Régnard JF, Burroni B, Goc J, Biton J, Ouakrim H, Cremer I, Dieu-Nosjean MC and Damotte D: Systemic inflammation, nutritional statusand tumor immune microenvironment determine outcome of resected nonsmall cell lung cancer. Plos One 9: e106914, 2014.

12 Ignacio de Ulíbarri J, González-Madroño A, de Villar NG, González P, González B, Mancha A, Rodríguez F and Fernández G: CONUT: A tool for controlling nutritional status. First validation in a hospital population. Nutr Hosp 20: 38-45, 2005.

13 de Ulibarri Perez JI, Femandez G, Rodriguez Salvanes F and Diaz Lopez AM: Nutritional screening control of clinical undernutrition with analytical parameters. Nutr Hosp 29: 797-811, 2014.

14 Ueno T, Hirayama S, Ito M, Nishioka E, Fukushima Y, Satoh T, Idei M, Horiuchi Y, Shoji H, Ohmura H, Shimizu T and Miida T: Albumin concentration determined by the modified bromocresol purple method is superior to that by the bromocresol green method for assessing nutritional status in malnourished patients with inflammation. Ann Clin Biochem 50: 576-584, 2013.

15 Nakagomi A, Kohashi K, Morisawa T, Kosugi M, Endoh I, Kusama Y, Atarashi H and Shimizu W: Nutritional status is associated with inflammation and predicts a poor outcome in patients with chronic heart failure. J Atheroscler Thromb 23: 713-712, 2016.

16 Narumi T, Arimoto T, Funayama A, Kadowaki S, Otaki Y, Nishiyama S, Takahashi H, Shishido T, Miyashita T, Miyamoto T, Watanabe T and Kubota I: Prognostic importance of objective nutritional indexes in patients with chronic heart failure. $J$ Cardiol 62: 307-313, 2013.

17 Taniguchi E, Kawaguchi T, Otsuka M, Uchida Y, Nagamatsu A, Itou M, Oriishi T, Ishii K, Imanaga M, Suetsugu T, Otsuyama J, Ibi R, Ono M, Tanaka S and Sata M: Nutritional assessments for ordinary medical care in patients with chronic liver disease. Hepatol Res 43: 192-199, 2013.

18 Lopez-Larramona G, Lucendo AJ and Tenias JM: Association between nutritional screening via the controlling nutritional status index and bone mineral density in chronic liver disease of various etiologies. Hepatol Res 45: 618-628, 2015.

19 Iseki Y, Shibutani M, Maeda K, Nagahara H, Ohtani H, Sugano K, Ikeya T, Muguruma K, Tanaka H, Toyokawa T, Sakurai K and Hirakawa $\mathrm{K}$ : Impact of the preoperative controlling nutritional status (CONUT) score on the survival after curative surgery for colorectal cancer. Plos One 10: e0132488, 2014. 
20 Hirahara N, Matsubara T, Hayashi H, Takai K, Nakada S and Tajima Y: Prognostic importance of controlling nutritional status in patients undergoing curative thoracoscopic esophagectomy for esophageal cancer. Am J Ther DOI:10.1097/MJT.0000000 $000000414,2016$.

21 Varlotto JM, Recht A, Flickinger JC, Medford-Davis LN, Dyer AM and DeCamp MM: Factors associated with local and distant recurrence and survival in patients with resected nonsmall cell lung cancer. Cancer 115: 1059-1069, 2009.

22 Goldstraw P, Crowley J, Chansky K, Giroux DJ, Groome PA, Rami-Porta R, Postmus PE, Rusch V and Sobin L; International Association for the Study of Lung Cancer International Staging Committee.; Participating Institutions. The IASLC lung cancer staging project: Proposal for the revision of the TNM stage groupings in the forthcoming (seventh) edition of the TNM Classification of Malignant Tumours. J Thorac Oncol 2: 706714, 2007.

23 Shoji F, Morodomi Y, Akamine T, Takamori S, Katsura M, Takada K, Suzuki Y, Fujishita T, Okamoto T and Maehara Y: Predictive impact for postoperative recurrence using the preoperative prognostic nutritional index in pathological stage I non-small cell lung cancer. Lung Cancer 98: 15-21, 2016.
24 Onodera T, Goseki N and Kosaki G: Prognostic nutritional index in gastrointestinal surgery of malnourished cancer patients. Nihon Geka Gakkai Zasshi 85: 1001-1005, 1984.

25 Cengiz O, Kocer B, Surmeli S, Santicky MJ and Soran A: Are pretreatment serum albumin and cholesterol levels prognostic tools in patients with colorectal carcinoma? Med Sci Monit 12: 240-247, 2006.

26 Ko Y, Park YH, Lee JW, Ku JH, Kwak C and Kim HH: Influence of nutritional deficiency on prognosis of renal cell carcinoma. BJU Int 112: 775-780, 2013.

27 Cubiella J, Castells A, Fondevila C, Sans M, Sabater L, Navarro $\mathrm{S}$ and Fernández-Cruz L: Prognostic factors in nonresectable pancreatic adenocarcinoma: a rationale to design therapeutic trials. Am J Gastroenterol 94: 1271-1278, 1999.

Received November 22, 2016

Revised January 6, 2017

Accepted January 19, 2017 